\title{
Phase response analysis during in vivo-like high conductance states; dendritic SK determines the mean and variance of responses to dendritic excitation
}

\author{
Nathan W Schultheiss ${ }^{1 *}$, Jeremy R Edgerton ${ }^{2}$, Dieter Jaeger ${ }^{2}$ \\ From Nineteenth Annual Computational Neuroscience Meeting: CNS*2010 \\ San Antonio, TX, USA. 24-30 July 2010
}

A neuron's phase response curve (PRC) describes how synaptic inputs at different times during the spike cycle affect the timing of subsequent spikes, and PRC analysis is a powerful technique for predicting and interpreting the emergence of synchronous modes in synaptically coupled networks and neuronal populations receiving common input. However, neuronal PRCs are typically measured during intrinsic pacemaking which may not reflect neuronal excitability and dynamics during high conductance states generated by complex network activity in vivo. Using a full morphological model of a globus pallidus (GP) neuron we have recently demonstrated that during intrinsic pacemaking, somatic PRCs for GP neurons are type I, i.e. excitatory inputs at all phases of the spike cycle advance the spontaneous spiking rhythm ${ }^{1}$. We also demonstrated that synaptic excitation of the distal dendrite can paradoxically delay subsequent spiking when delivered at some phases of the spike cycle (yielding a type II PRC) as a consequence of dendritic activation of the small conductance calcium-activated potassium current, $\mathrm{SK}^{1}$. Since during high conductance states spike timing is determined by a balance between intrinsic mechanisms and synaptic input fluctuations, in this study we investigated how somatic and dendritic phase response properties of the GP model are affected by ongoing stochastic synaptic background activity.

We generated high conductance states in the model by applying synaptic backgrounds composed of randomly-timed excitatory and inhibitory synaptic inputs at 1022 GABA synapses and 100 AMPA synapses

* Correspondence: nschult@bu.edu

${ }^{1}$ Department of Psychology, Boston University, Boston, MA 02215, USA distributed throughout the dendrite. By varying the synaptic gain and input frequency parameters across the physiological range for inputs to GP, we achieved a diverse set of high conductance states characterized by sub-threshold voltage fluctuations, irregular spiking, and elevated membrane conductance (Figure 1A \&1B) and spanning the range of spike frequencies observed in vivo $(15-45 \mathrm{~Hz})$. For each synaptic background parameter set, we generated 100 single-trial PRCs by delivering a single $2.5 \mathrm{nS}$ AMPA-synaptic input to either the soma or distal dendrite at each of 72 time-points (in separate simulations) within the first spike cycle of each of 100 control spike trains (Figure 1C \&1D). We then averaged the single-trial PRCs for each synaptic background to evaluate the dependence of PRC shape on stimulus location, spike frequency, and dendritic SK conductance, in addition to the gain and input frequencies of synaptic backgrounds. Next, we analyzed on a trial-by-trial basis the interactions of PRC stimuli with transient fluctuations in the synaptic background leading to added or skipped spikes. We determined that dendritic SK underlies both the incidence of skipped spikes and the dependence of skipped spike events on stimulus phase. Interestingly, the input-phase dependence of skipped spike events mirrored the phase-dependent variance in the PRC itself (which we plotted as phase response-variance curves, PRVCs). This indicates that dendritic SK determines not only the mean response, but also the variance of responses, to excitatory dendritic inputs. Average somatic and dendritic PRCs were type I and type II, respectively, and are likely to represent the average behavior of populations of GP neurons in response to shared excitation. Furthermore, in-as-much as the GP 


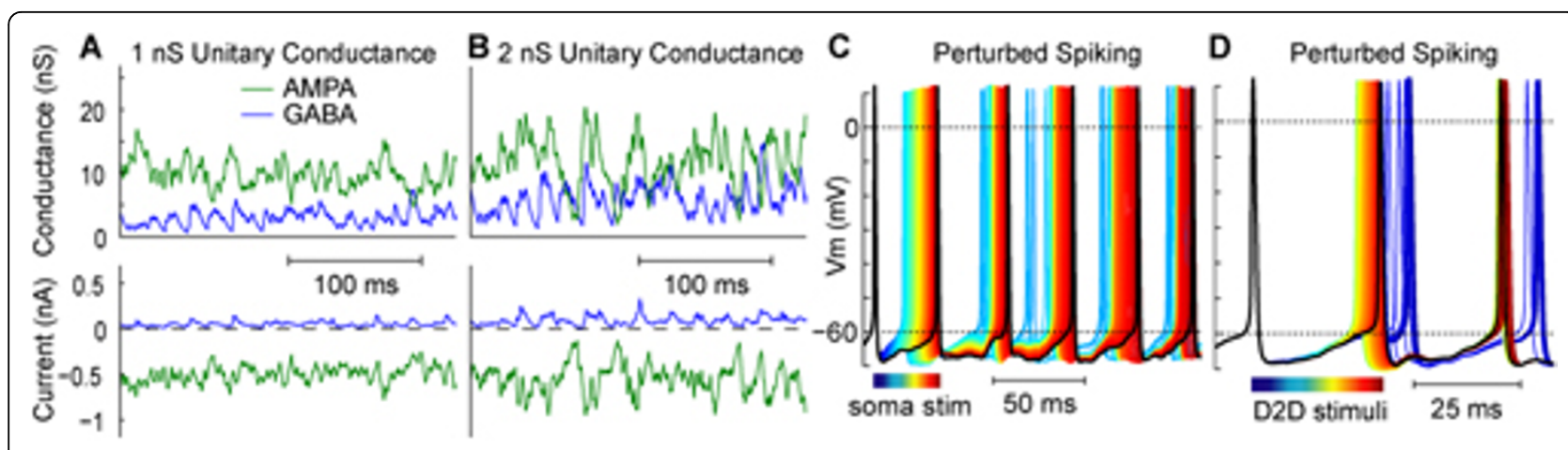

Figure 1 A\&B. Total synaptic conductance (top) and current (bottom) for synaptic backgrounds composed of $1 \mathrm{nS}$ (A) or $2 \mathrm{nS}$ (B) unitary inputs. C\&D. Perturbations of control spike trains (black) by somatic (C) or dendritic (D2D)(D) stimuli. (Trace color indicates input phase.)

model and simulated synaptic backgrounds are physiologically realistic, the variance in the model's responses across trials directly reflects a major source of spike time variance across populations of GP neurons in vivo.

\section{Author details}

'Department of Psychology, Boston University, Boston, MA 02215, USA.

${ }^{2}$ Department of Biology, Emory University, Atlanta, GA 30322, USA.

Published: 20 July 2010

\section{Reference}

1. Schultheiss NW, Edgerton JR, Jaeger D: Phase response curve analysis of a full morphological globus pallidus neuron model reveals distinct perisomatic and dendritic modes of synaptic integration. J Neurosci 2010, 30:2767-2782.

doi:10.1186/1471-2202-11-S1-012

Cite this article as: Schultheiss et al:: Phase response analysis during in vivo-like high conductance states; dendritic SK determines the mean and variance of responses to dendritic excitation. BMC Neuroscience 2010 11(Suppl 1):012.
Submit your next manuscript to BioMed Central and take full advantage of:

- Convenient online submission

- Thorough peer review

- No space constraints or color figure charges

- Immediate publication on acceptance

- Inclusion in PubMed, CAS, Scopus and Google Scholar

- Research which is freely available for redistribution 